\title{
The Lojasiewicz exponent over a field of arbitrary characteristic
}

\author{
Szymon Brzostowski • Tomasz Rodak
}

Received: 11 March 2014 / Accepted: 5 December 2014 / Published online: 13 January 2015

(C) The Author(s) 2015. This article is published with open access at Springerlink.com

\begin{abstract}
Let $\mathbb{K}$ be an algebraically closed field and let $\mathbb{K}\left(\left(X^{\mathbb{Q}}\right)\right)$ denote the field of generalized series with coefficients in $\mathbb{K}$. We propose definitions of the local Łojasiewicz exponent of $F=\left(f_{1}, \ldots, f_{m}\right) \in \mathbb{K}[[X, Y]]^{m}$ as well as of the Łojasiewicz exponent at infinity of $F=\left(f_{1}, \ldots, f_{m}\right) \in \mathbb{K}[X, Y]^{m}$, which generalize the familiar case of $\mathbb{K}=\mathbb{C}$ and $F \in \mathbb{C}\{X, Y\}^{m}$ (resp. $F \in \mathbb{C}[X, Y]^{m}$ ), see Chądzyński and Krasiński (In: Singularities, 1988; In: Singularities, 1988; Ann Polon Math 67(3):297-301, 1997; Ann Polon Math 67(2):191-197, 1997), and prove some basic properties of such numbers. Namely, we show that in both cases the exponent is attained on a parametrization of a component of $F$ (Theorems 6 and 7), thus being a rational number. To this end, we define the notion of the Łojasiewicz pseudoexponent of $F \in\left(\mathbb{K}\left(\left(X^{\mathbb{Q}}\right)\right)[Y]\right)^{m}$ for which we give a description of all the generalized series that extract the pseudoexponent, in terms of their jets. In particular, we show that there exist only finitely many jets of generalized series giving the pseudoexponent of $F$ (Theorem 5). The main tool in the proofs is the algebraic version of Newton's Polygon Method. The results are illustrated with some explicit examples.
\end{abstract}

Keywords Generalized power series · Łojasiewicz exponent · Parametrization · Newton polygon method

The paper was partially supported by the Polish National Science Centre (NCN) Grants No. 2012/07/B/ST1/03293 and 2013/09/D/ST1/03701.

\section{S. Brzostowski · T. Rodak $(\varangle)$}

Faculty of Mathematics and Computer Science,

University of Łódź, ul. Banacha 22, 90-238 Łódź, Poland

e-mail: rodakt@math.uni.lodz.pl

S. Brzostowski

e-mail: brzosts@math.uni.lodz.pl 
Mathematics Subject Classification $13 \mathrm{~F} 25 \cdot 14 \mathrm{~B} 05 \cdot 32 \mathrm{~S} 10$

\section{Introduction}

Let $f:\left(\mathbb{R}^{n}, \mathbf{0}\right) \rightarrow(\mathbb{R}, 0)$ be a real analytic function. The Łojasiewicz Inequality asserts that there exist $v, C>0$ such that

$$
|f(x)| \geqslant C \operatorname{dist}(x, V(f))^{v}, \quad x \text { near } \mathbf{0},
$$

where $V(f)$ stands for the zero set of $f$. The problem is to determine the smallest possible exponent $v$ in (1). It is known that this exponent is rational [4] and equal to the rate of growth of $f$ on some analytic path centered near the origin [19]. In the particular two-dimensional case the optimal exponent $v$ can be expressed in terms of the Puiseux roots of $f$ [14].

Now, let $F:\left(\mathbb{C}^{n}, \mathbf{0}\right) \rightarrow\left(\mathbb{C}^{m}, \mathbf{0}\right)$ be an analytic map with an isolated zero at the origin. In this case a counterpart of the problem described above is to find an optimal exponent in the inequality

$$
|F(z)| \geqslant C|z|^{v}
$$

where $C$ is a positive constant and $z$ is in a sufficiently small neighbourhood of $\mathbf{0}$. This exponent is called the local Łojasiewicz exponent of $F$ and is denoted by $\mathfrak{L}_{0}(F)$. Again it is known that it is a rational number and

$$
\mathfrak{L}_{0}(F)=\sup _{\Phi} \frac{\operatorname{ord} F \circ \Phi}{\operatorname{ord} \Phi},
$$

where $\Phi$ runs through the set of all analytic paths centered in $\mathbf{0} \in \mathbb{C}^{n}$. Moreover, if $F$ is a regular sequence (i.e. $n=m$ ), then for generic direction $\ell \in \mathbb{P}^{n-1}$ the exponent $\mathfrak{L}_{0}(F)$ is attained on the curve $F^{-1}(\ell)$ (see [15] or [16] for a different proof of this result). Another observation of this kind is the following

Theorem $1([8,10])$ Let $F:=\left(f_{1}, \ldots, f_{m}\right), S:=\left\{f_{1} \times \cdots \times f_{m}=0\right\}$. Then

$$
\mathfrak{L}_{0}(F)=\inf \left\{\nu \in \mathbb{R}: \exists_{\varepsilon, C>0} \forall_{z \in S}|z|<\varepsilon \Rightarrow|F(z)| \geqslant C|z|^{\nu}\right\} .
$$

In particular, if $F \neq \mathbf{0}$ and $n=2$ then the local Łojasiewicz exponent of $F$ is attained on one of the curves $\left\{f_{i}=0\right\}$.

In other words,

$$
\mathfrak{L}_{0}(F)=\frac{\operatorname{ord}_{t} F(\Phi(t))}{\operatorname{ord}_{t} \Phi(t)},
$$

where $\Phi(t) \in \mathbb{C}\{t\}^{n} \backslash\{\mathbf{0}\}, \Phi(0)=\mathbf{0}$ and $f_{i}(\Phi(t))=0$ for some non-zero $f_{i}$. 
If $F: \mathbb{C}^{n} \rightarrow \mathbb{C}^{m}$ is a polynomial map with finite number of zeroes, then it is also possible to define so-called Łojasiewicz exponent of $F$ at infinity (or global Łojasiewicz exponent of $F$ ). Namely, we are looking for the greatest exponent $v$ in the inequality (2), where $C$ is a positive constant and $z$ is outside a sufficiently big ball. This optimal exponent is called the Lojasiewicz exponent of $F$ at infinity and is denoted by $\mathfrak{L}_{\infty}(F)$. Similarly as in the local case, this exponent is rational and is attained on a curve centered at infinity. More precisely, there exists a meromorphic map $\Phi: \mathbb{E} \backslash\{0\} \rightarrow \mathbb{C}^{n}$, where $\mathbb{E}$ is the unit ball in $\mathbb{C}$, such that $\lim _{t \rightarrow 0} \Phi(t)=\infty$ and $\mathfrak{L}_{\infty}(F)$ is equal to the rate of growth of $F$ on the image of $\Phi$. Thus, we may write

$$
\mathfrak{L}_{\infty}(F)=\inf _{\Phi} \frac{\operatorname{ord}_{t} F(\Phi(t))}{\operatorname{ord}_{t} \Phi(t)},
$$

where $\Phi$ is as above and, in fact, the infimum is just the minimum. Moreover, the following theorem holds:

Theorem $2([7,11])$ Let $F:=\left(f_{1}, \ldots, f_{m}\right), S:=\left\{f_{1} \times \cdots \times f_{m}=0\right\}$. Then

$$
\mathfrak{L}_{\infty}(F)=\sup \left\{v \in \mathbb{R}: \exists_{R, C>0} \forall_{z \in S}|z|>R \Rightarrow|F(z)| \geqslant C|z|^{\nu}\right\} .
$$

In particular, if $F \neq \mathbf{0}$ and $n=2$ then there exists a meromorphic map $\Phi: \mathbb{E} \backslash\{0\} \rightarrow$ $\mathbb{C}^{n}$ such that $\lim _{t \rightarrow 0} \Phi(t)=\infty, f_{i} \circ \Phi=0$ for some non-zero $f_{i}$ and

$$
\mathfrak{L}_{\infty}(F)=\frac{\operatorname{ord}_{t} F(\Phi(t))}{\operatorname{ord}_{t} \Phi(t)} .
$$

The main goal of the paper is to show that in the above theorems, at least in the two dimensional case, one may replace the field $\mathbb{C}$ with an algebraically closed field of arbitrary characteristic. Namely, let $\mathbb{K}$ be an algebraically closed field. The formulas (3) and (4) provide the definitions of local and global Łojasiewicz exponents in $\mathbb{K}\left[\left[x_{1}, \ldots, x_{n}\right]\right]$ and in $\mathbb{K}\left[x_{1}, \ldots, x_{n}\right]$, respectively. Now, let $n=2$. In this setting, our main results are Theorems 6 and 7. They give direct two dimensional counterparts of the above-mentioned Theorems 1 and 2 .

Proofs of these theorems over $\mathbb{C}$ use metric properties of the field or, in the two dimensional case, the Newton-Puiseux theorem, which is false in positive characteristic. Thus, we cannot apply these methods. Our idea is to introduce, with the help of the field of generalized series $\mathbb{K}\left(\left(X^{\mathbb{Q}}\right)\right)$, some auxiliary notion (called the Łojasiewicz. pseudoexponent $)$, which is, roughly speaking, the greatest vanishing order of the map $F=\left(f_{1}, \ldots, f_{m}\right) \in \mathbb{K}\left(\left(X^{\mathbb{Q}}\right)\right)[Y]^{m}$ on all paths of the form $(t, y(t)), y(t) \in \mathbb{K}\left(\left(t^{\mathbb{Q}}\right)\right)$. It turns out (see Theorem 5) that this number is rational (if finite) and, what is more important for us, it is the vanishing order of $F$ on a path $\left(t, y_{0}(t)\right)$, where $y_{0}(t)$ is a root of some $f_{j}$. Moreover, in this theorem we prove that all the paths on which the pseudoexponent is attained are similar to such $\left(t, y_{0}(t)\right)$ 's in the sense of jets (see Definition 6). Once Theorem 5 is proved, the only non-standard information needed to deduce Theorems 6 and 7 is Proposition 3. This proposition explains the relation between the valuations defined by two different types of parametrizations (namely the standard Hamburger-Noether and the generalized ones). 


\section{The abstract case of an arbitrary field}

In the case of a field $\mathbb{K}$ of characteristic 0 one can apply the Newton-Puiseux theorem to find the roots of an arbitrary $f \in \mathbb{K}((X))[Y]$ (a polynomial with coefficients in the Laurent series field) of positive degree. In short, $\overline{\mathbb{K}((t))}=\overline{\mathbb{K}}\left(\left(t^{*}\right)\right)$, where $\mathbb{K}\left(\left(t^{*}\right)\right)$ denotes the field of Puiseux series over the field $\mathbb{K}$. The same is true for fields $\mathbb{K}$ of positive characteristic $p$ but only under the additional assumption that $p \nmid \operatorname{deg}_{Y} f$ (see [2, Theorem 5.14]). Thus, in general, one needs to extend the field $\overline{\mathbb{K}}\left(\left(t^{*}\right)\right)$ even more to find the algebraic closure of $\mathbb{K}((t))$. We recall the following notion.

Definition 1 Let $\mathbb{K}$ be a field. By $\mathbb{K}\left(\left(t^{\mathbb{Q}}\right)\right)$ we will denote the field of all generalized series with coefficients in $\mathbb{K}$, that is formal sums of the form $u(t)=\sum_{q \in \mathbb{Q}} u_{q} t^{q}$, where $u_{q} \in \mathbb{K}$ and the support of $u(t), \operatorname{Supp}_{t} u(t):=\left\{q \in \mathbb{Q}: u_{q} \neq 0\right\}$, is a well-ordered set.

The fact that the support of every element of $\mathbb{K}\left(\left(t^{\mathbb{Q}}\right)\right)$ forms a well-ordered set implies that $\mathbb{K}\left(\left(t^{\mathbb{Q}}\right)\right)$ is indeed a field (with the natural definitions of addition and multiplication), an overfield of $\mathbb{K}\left(\left(t^{*}\right)\right)$. But even more is true.

Theorem 3 ([18, Theorem 5.2]) The algebraic closure of the field $\mathbb{K}\left(\left(t^{\mathbb{Q}}\right)\right)$ is equal to $\overline{\mathbb{K}}\left(\left(t^{\mathbb{Q}}\right)\right)$.

Although the field $\overline{\mathbb{K}}\left(\left(t^{\mathbb{Q}}\right)\right)$ is algebraically closed, it is much bigger than the actual algebraic closure of $\mathbb{K}((t))$. The precise description of $\overline{\mathbb{K}((t))}$ was given by $\mathrm{K}$. Kedlaya in [13], but we will make no use of this description, working entirely in the larger field $\overline{\mathbb{K}}\left(\left(t^{\mathbb{Q}}\right)\right)$.

An alternative way of parametrizing the "zero set" of an $f \in \mathbb{K}[[X, Y]]$ of positive order is by utilizing so-called Hamburger-Noether expansions. More precisely, the following holds.

Theorem 4 (cf. [6,17]) Let $\mathbb{K}=\overline{\mathbb{K}}, f \in \mathbb{K}[[X, Y]], f(\mathbf{0})=0$. Then there exists a pair $(\varphi(t), \psi(t)) \in \mathbb{K}[[t]]^{2} \backslash\{\mathbf{0}\}$ with $\varphi(0)=\psi(0)=0$, such that

$$
f(\varphi(t), \psi(t))=0 \text {. }
$$

Conversely, for any pair $(\varphi(t), \psi(t))$ as above there exists an $f \in \mathbb{K}[[X]][Y]$, irreducible as an element of $\mathbb{K}[[X, Y]]$, with $f(\mathbf{0})=0$ and such that

$$
f(\varphi(t), \psi(t))=0
$$

The above theorem will also be extended to the case of a pair $(\varphi(t), \psi(t)) \in$ $\mathbb{K}((t))^{2}$ (Proposition 2 below). Anyway, the discussion above motivates the following definition (cf. also Definition 8).

Definition 2 Let $\mathbb{K}$ be a field. Any pair of the form $(t, y(t))$ with $y \in \mathbb{K}\left(\left(t^{\mathbb{Q}}\right)\right)$ (resp. $(\varphi(t), \psi(t)) \in \mathbb{K}[[t]]^{2} \backslash\{\mathbf{0}\}, \varphi(0)=\psi(0)=0$ ) will be called a generalized (resp. formal) parametrization. We will say that such a pair is a generalized (resp. 
formal) parametrization of $f$ iff $f(t, y(t))=0$ (resp. $f(\varphi(t), \psi(t))=0$ ), where $f \in \mathbb{K}\left(\left(X^{\mathbb{Q}}\right)\right)[Y]$ (resp. $\left.f \in \mathbb{K}[[X, Y]]\right)$.

We state now the natural generalizations of the classical definitions of the (local and at infinity) Łojasiewicz exponents. Namely, we adapt (3) and (4) as the defining conditions allowing $\Phi$ to run through the set of all formal parametrizations (resp. parametrizations at infinity-cf. Definition 8).

Definition 3 Let $\mathbb{K}$ be a field. For an $F \in \mathbb{K}[[X, Y]]^{m}$ with $F(\mathbf{0})=\mathbf{0}$ we define the local Łojasiewicz exponent of $F$ as the number (or $+\infty)$ given by ${ }^{1}$

$$
\mathfrak{L}_{0}(F):=\sup _{\substack{\Phi \in \overline{\mathbb{K}}[[t]]^{2} \\ 0<\operatorname{ord} \Phi<\infty}} \frac{\operatorname{ord}_{t} F \circ \Phi}{\operatorname{ord}_{t} \Phi} .
$$

Similarly, for an $F \in \mathbb{K}[X, Y]^{m}$ we define the Łojasiewicz exponent of $F$ at infinity or the global Łojasiewicz exponent of $F$ as the number (or $-\infty$ ) given by

$$
\mathfrak{L}_{\infty}(F):=\inf _{\substack{\Phi \in \overline{\mathbb{K}}((t))^{2} \\ \operatorname{ord} \Phi<0}} \frac{\operatorname{ord}_{t} F \circ \Phi}{\operatorname{ord}_{t} \Phi} .
$$

The main tool in the paper is the following notion of the Łojasiewicz pseudoexponent.

Definition 4 Let $\mathbb{K}$ be a field and let $F \in \mathbb{K}\left(\left(X^{\mathbb{Q}}\right)\right)[Y]^{m}$. The (Lojasiewicz) pseudoexponent of $F$ is the number (or $+\infty$ )

$$
\overline{\mathfrak{L}}_{Y}(F):=\sup _{y(t) \in \overline{\mathbb{K}}\left(\left(t^{\mathbb{Q}}\right)\right)} \operatorname{ord}_{t} F(t, y(t)) .
$$

Note that the value of $\overline{\mathfrak{L}}_{Y}(F)$ depends on the roles played by the variables $X$ and $Y$, however, as long as no confusion is likely, we will simply write $\overline{\mathfrak{L}}(F)$ instead of $\overline{\mathfrak{L}}_{Y}(F)$.

Remark 1 The above definitions can also be stated more generally - one can consider the exponents with respect to an intermediate field $\mathbb{L}$ such that $\mathbb{K} \subset \mathbb{L} \subset \overline{\mathbb{K}}$. For example, $\mathbb{L}$ could be a real closed field. We will not dive into this topic here.

\section{Auxiliary results}

\subsection{Newton's polygon method}

We recall that for a non-zero series $z \in \mathbb{K}\left(\left(t^{\mathbb{Q}}\right)\right)$ of the form $z(t)=\sum_{q \in \mathbb{Q}} z_{q} t^{q}$ there are defined:

\footnotetext{
1 Throughout this paper, ord of a tuple means the minimal ord of its components.
} 
- Its order $\operatorname{ord}_{t} z:=\min (\operatorname{Supp}(z))$,

- Its initial coefficient inco $z:=z_{\operatorname{Ord}_{t} z}$,

- Its initial form info $t z:=$ inco $_{t} z \cdot t^{\operatorname{ord}_{t} z}$.

Moreover, $\operatorname{ord}_{t} 0:=\infty$, inco $_{t} 0:=0, \operatorname{info}_{t} 0:=0$.

Following Abhyankar [2] we will use the symbol $\ominus$ to denote an unspecified (anonymous) non-zero element of a field under consideration.

Definition 5 Let $z(t) \in \mathbb{K}\left(\left(t^{\mathbb{Q}}\right)\right), z(t)=\sum_{q \in \mathbb{Q}} z_{q} t^{q}$, let $U$ be an indeterminate over $\mathbb{K}\left(\left(t^{\mathbb{Q}}\right)\right)$ and let $\mathbb{L}$ be an overfield of $\mathbb{K}(U)$. We say that a series $v(t) \in \mathbb{L}\left(\left(t^{\mathbb{Q}}\right)\right)$ is a $(Q, U)$-deformation of $z(t)$, if $Q \in \mathbb{Q}$ and

$$
\operatorname{info}_{t}(v(t)-z(t))=\left(U-z_{Q}\right) t^{Q} .
$$

In other words, any series of the form $v(t)=\sum_{q \in \mathbb{Q}} v_{q} t^{q}$ where

$-v_{q}=z_{q}$ for $q<Q$,

$-v_{Q}=U$,

- $v_{q} \in \mathbb{L}$ for $q>Q$,

is a $(Q, U)$-deformation of $z(t)$.

We begin with an algebraic restatement of Newton's Polygon Method. It is a simplified but generalized version of [2, Theorem 14.2], see also [5].

Proposition 1 (Newton's polygon method) Let $\mathbb{K}$ be a field and let $g \in \mathbb{K}\left(\left(X^{\mathbb{Q}}\right)\right.$ ) $[Y] \backslash\{0\}$. Write

$$
g(X, Y)=e(X) \prod_{1 \leqslant j \leqslant k}\left(Y-z^{j}(X)\right) \quad \text { with } e(X) \in \mathbb{K}\left(\left(X^{\mathbb{Q}}\right)\right)
$$

where $z^{j}(X) \in \overline{\mathbb{K}}\left(\left(X^{\mathbb{Q}}\right)\right)$ for $1 \leqslant j \leqslant k(g(X, Y)=e(X) \neq 0$ allowed $)$.

Let $u(t):=\sum_{\substack{q \leqslant Q \\ q \in \mathbb{Q}}} u_{q} t^{q} \in \overline{\mathbb{K}}\left(\left(t^{\mathbb{Q}}\right)\right)$, where $Q \in \mathbb{Q}$, and let $v(t)$ be any $(Q, U)$ deformation of $0 \in \overline{\mathbb{K}}\left(\left(t^{\mathbb{Q}}\right)\right)$. Then

$\rightarrow \operatorname{info}_{t} g(t, u(t)+v(t))$ is independent of the particular choice of the deformation $v(t)$

$\rightarrow$ For $h:=\operatorname{inco}_{t} g(t, u(t)+v(t))$ it is $h \in \overline{\mathbb{K}}[U] \backslash\{0\}$ (and even $h \in \mathbb{K}\left(u_{q}: q \leqslant\right.$ $Q)[U])$

$\rightarrow$ The following two conditions are equivalent:

(i) There exists $1 \leqslant j_{0} \leqslant k$ such that $\operatorname{ord}_{t}\left(u(t)-z^{j_{0}}(t)\right)>Q$

(ii) The polynomial $h$ vanishes for $U=0$

The following two conditions are equivalent:

(iii) For every $1 \leqslant j \leqslant k$ it is $\operatorname{ord}_{t}\left(u(t)-z^{j}(t)\right)<Q$

(iv) The polynomial $h$ is constant 
What is more,

- If $U=0$ is of multiplicity $l>0$ as a root of $h$, then there exist exactly $l$ different indices $j_{1}, \ldots, j_{l} \in\{1, \ldots, k\}$ for which

$$
\operatorname{ord}_{t}\left(u(t)-z^{j_{i}}(t)\right)>Q, \quad i=1, \ldots, l
$$

- If $\operatorname{deg}_{U} h=m>0$, then there exist exactly $m$ different indices $j_{1}, \ldots, j_{m} \in$ $\{1, \ldots, k\}$ for which

$$
\operatorname{ord}_{t}\left(u(t)-z^{j_{i}}(t)\right) \geqslant Q, \quad i=1, \ldots, m
$$

Proof It is easy to see that all the assertions of the theorem can be obtained from the particular case $g(X, Y)=e(X)(Y-z(X))$, where $0 \neq e(X) \in \mathbb{K}\left(\left(X^{\mathbb{Q}}\right)\right), z(X) \in$ $\overline{\mathbb{K}}\left(\left(X^{\mathbb{Q}}\right)\right)$ (the theorem being obvious for $\left.g(X, Y)=e(X)\right)$. However, for such a $g$ and any $(Q, U)$-deformation $v(t)$ of $0 \in \overline{\mathbb{K}}\left(\left(t^{\mathbb{Q}}\right)\right)$ we can take $r:=\operatorname{ord}_{t}(u(t)-z(t))$ and $s:=\operatorname{ord}_{t} e(t)$ to obtain

$$
\begin{aligned}
& \operatorname{info}_{t} g(t, u(t)+v(t)) \\
& =\operatorname{info}_{t}(e(t)(u(t)+v(t)-z(t)))=\ominus \begin{cases}\ominus t^{r+s}, & \text { if } r<Q \\
(U+\ominus) t^{Q+s}, & \text { if } r=Q \\
U t^{Q+s}, & \text { if } r>Q\end{cases} \\
& =\ominus\left(\delta_{Q}^{\min (r, Q)} U+\ominus \delta_{Q}^{\max (r, Q)}\right) \cdot t^{\min (r, Q)+s},
\end{aligned}
$$

where $\delta$ is the Kronecker delta, and the $\ominus \in \overline{\mathbb{K}}$ are independent of the choice of $v(t)$ as they are determined by the coefficients of $u(t)-z(t)$ of order $\leqslant Q$. Hence, $h=$ inco $_{t} g(t, u(t)+v(t)) \in \overline{\mathbb{K}}[U]$ and $h(0)=0$ iff $r>Q$, which gives "(i) $\Leftrightarrow$ (ii)". Similarly, $h$ is constant iff $r<Q$ so "(iii) $\Leftrightarrow$ (iv)". The last two assertions are obvious.

We also remark that for a general $g \in \mathbb{K}\left(\left(X^{\mathbb{Q}}\right)\right)[Y]$ the fact that $h \in \mathbb{K}\left(u_{q}: q \leqslant\right.$ $Q)[U]$ follows immediately, since in particular $h=$ inco $_{t} g\left(t, u(t)+U t^{Q}\right)$.

Example 1 Let $g(X, Y):=Y^{p}-X^{p-1} Y-X^{p-1} \in \mathbb{K}[X, Y]$, where $\mathbb{K}$ is a field of positive characteristic $p$. Then, following [1], we may write

$$
g(X, Y)=\prod_{j=0}^{p-1}\left(Y-j X-\sum_{k=1}^{\infty} X^{1-p^{-k}}\right) .
$$

Put $u(t):=\sum_{k=1}^{\infty} t^{1-p^{-k}}$. Then $g(t, u(t))=0$. Let $v(t):=U t+\cdots$ be a $(1, U)$ deformation of $0 \in \mathbb{K}\left(\left(t^{\mathbb{Q}}\right)\right)$. We have $g(t, u(t)+v(t))=U\left(U^{p-1}-1\right) t^{p}+\cdots$ and consequently $h(U)=$ inco $_{t} g(t, u(t)+v(t))=U\left(U^{p-1}-1\right)$.

\subsection{Jets and truncations}

Definition 6 Let $q \in \mathbb{Q}$ and let us treat $\mathbb{K}\left(\left(t^{\mathbb{Q}}\right)\right)$ as a $\mathbb{K}\left[\left[t^{\mathbb{Q}}\right]\right]$-module. We define the $q$-th order open jet space $\stackrel{\circ}{\mathscr{J}} q=\stackrel{\mathscr{J}}{q}\left[\mathbb{K}\left(\left(t^{\mathbb{Q}}\right)\right)\right]$ of $\mathbb{K}\left(\left(t^{\mathbb{Q}}\right)\right)$ as the module 
$\mathbb{K}\left(\left(t^{\mathbb{Q}}\right)\right) /\left(t^{q}\right)$ and $q$-th order closed jet space $\mathscr{J}^{q}=\mathscr{J}^{q}\left[\mathbb{K}\left(\left(t^{\mathbb{Q}}\right)\right)\right]$ of $\mathbb{K}\left(\left(t^{\mathbb{Q}}\right)\right)$ as the module

$$
\mathbb{K}\left(\left(t^{\mathbb{Q}}\right)\right) / \bigcup_{\substack{r>q \\ r \in \mathbb{Q}}}\left(t^{r}\right) .
$$

The union of the two types of $q$-th order jet spaces will be denoted by $\mathbb{J}^{q}=$ $\mathbb{J}^{q}\left[\mathbb{K}\left(\left(t^{\mathbb{Q}}\right)\right)\right]$.

Note that unlike e.g. the smooth functions case, the jet spaces defined above do not constitute rings (the multiplication is not associative).

The elements of $\mathscr{J}^{q}$ are called ( $q$-th order) closed jets. A closed jet determined by a series $v$ will be denoted by $\mathscr{J}^{q}[v]$. For any set $\mathscr{S}$ of generalized series the set of closed jets $\mathscr{J}^{q}[\mathscr{S}]$ is defined in the obvious way. Similarly, the elements of $\dot{J}^{q}$ are called ( $q$-th order) open jets. An open jet determined by a series $v$ will be denoted by $\stackrel{\circ}{\mathscr{J}} q[v]$. For any set $\mathscr{S}$ of generalized series the set of open jets $\dot{J}^{q} q[\mathscr{S}]$ is defined in the obvious way. Similarly, there is defined the set of jets $\mathbb{J}^{q}[\mathscr{S}]:=\mathscr{J}^{q}[\mathscr{S}] \cup \mathscr{J}^{q}[\mathscr{S}]$.

Notation 1 For a closed jet $\iota=\mathscr{J}^{q}[\varphi]$ the symbol $i=\left(\mathscr{J}^{q}[\varphi]\right)$ will denote the interior of $\iota$ i.e. the jet $\stackrel{J}{J}^{q}[\varphi]$. Similarly, for any set $\mathfrak{A}$ of closed jets we put $\mathfrak{A}:=\{i$ : $\iota \in \mathfrak{A}\}$.

The jets have canonical representatives of the form $\sum_{s \leqslant q} a_{s} t^{s} \in \mathbb{K}\left(\left(t^{\mathbb{Q}}\right)\right.$ ) (or $\sum_{s<q} a_{s} t^{s} \in \mathbb{K}\left(\left(t^{\mathbb{Q}}\right)\right)$ in the case of open jets), nevertheless we find it useful to distinguish these objects from one another.

Definition 7 Let $\varphi \in \mathbb{K}\left(\left(t^{\mathbb{Q}}\right)\right)$ and $q \in \mathbb{Q}$. If $\varphi=\sum_{s \in \mathbb{Q}} \varphi_{s} t^{s}$ then we define the $q$-th order closed truncation $\varphi^{\leqslant q}$ of $\varphi$ as $\varphi \leqslant q(t):=\sum_{s \leqslant q} \varphi_{s} t^{s}$ and the $q$-th order open truncation $\varphi^{<q}$ of $\varphi$ as $\varphi^{<q}(t):=\sum_{s<q} \varphi_{s} t^{s}$. Thus $\varphi^{\leqslant q} \in \mathscr{J}^{q}[\varphi]$ and $\varphi^{<q} \in$ $\stackrel{\circ}{\mathscr{J}} q[\varphi]$.

In the following, all the formulas involving truncations are to be understood in the usual way (i.e. at the series level) while the formulas concerning jets are to be understood as representative-independent (i.e. valid at the jet level), for example this is the case with the formulas of the type $\operatorname{ord}_{t} g(t, \iota)$, where $g \in \mathbb{K}\left(\left(X^{\mathbb{Q}}\right)\right)[Y]$ and $\iota \in \mathbb{J}^{q}$.

Lemma 1 Let $\mathbb{K}$ be a field and $m \geqslant 2$. For any $m$-tuple $F \in \mathbb{K}\left(\left(X^{\mathbb{Q}}\right)\right)[Y]^{m}$ of coprime polynomials with $\operatorname{deg}_{Y} F>0$ and any $\varphi \in \mathbb{K}\left(\left(t^{\mathbb{Q}}\right)\right)$ the set $\mathscr{A}:=\{q \in \mathbb{Q}$ : $\left.\operatorname{ord}_{t} F\left(t, \varphi(t)+U t^{q}\right)=\operatorname{ord}_{t} F(t, \varphi(t))\right\}$ is non-empty. Moreover, there exists $\min \mathscr{A}$ and it is rational.

Proof Replacing $F(X, Y)$ with $F(X, \varphi(X)+Y)$ we may assume that $\varphi(t)=0$. Let $F=\left(f_{1}, \ldots, f_{m}\right)$, where

$$
f_{i}(X, Y)=a_{i 0}(X) Y^{d_{i}}+\cdots+a_{i d_{i}}(X), \quad a_{i 0}(X) \neq 0, i=1, \ldots, m .
$$


Since $f_{1}, \ldots, f_{m}$ are co-prime, $\min _{i} \operatorname{ord}_{t} a_{i d_{i}}(t)=\operatorname{ord}_{t} F(t, 0) \in \mathbb{Q}$. On the other hand, $\operatorname{ord}_{t} F\left(t, U t^{q}\right)=\min _{i, j}\left(\operatorname{ord}_{t} a_{i j}(t)+q\left(d_{i}-j\right)\right)$. Thus,

$$
\mathscr{A}=\left\{q \in \mathbb{Q}: \min _{i, j}\left(\operatorname{ord}_{t} a_{i j}(t)+q\left(d_{i}-j\right)\right)=\min _{i} \operatorname{ord}_{t} a_{i d_{i}}(t)\right\}
$$

Clearly, $\mathscr{A} \neq \varnothing$. Moreover, since $d_{i}>0$ for some $i$, we infer that there exists min $\mathscr{A}$.

Lemma 2 Let $\mathbb{K}$ be an infinite field. Let $F \in \mathbb{K}\left(\left(X^{\mathbb{Q}}\right)\right)[Y]^{m}, w \in \mathbb{K}\left(\left(t^{\mathbb{Q}}\right)\right)$ and $q \in \mathbb{Q}$. Then for every $(q, U)$-deformation $v(t)$ of $w(t)$ we have

$$
\operatorname{ord}_{t} F\left(t, w(t)+U t^{q}\right)=\operatorname{ord}_{t} F(t, v(t)) \text {. }
$$

Moreover, for any $N \in \mathbb{Q}$ the following conditions are equivalent:

1. $\operatorname{ord}_{t} F\left(t, w(t)+U t^{q}\right) \geqslant N$,

2. $\operatorname{ord}_{t} F(t, \varphi(t)) \geqslant N$, for every representative $\varphi(t)$ of $\stackrel{\circ}{\mathscr{J}} q[w(t)]$.

Proof As in the proof of the previous lemma, we may assume that $w(t)=0$. Moreover, it is sufficient to prove only the case $m=1$ and $F \neq \mathbf{0}$. Write

$$
F(X, Y)=a_{0}(X) Y^{d}+\cdots+a_{d}(X) .
$$

We have

$$
\operatorname{ord}_{t} F\left(t, U t^{q}\right)=\min _{j}\left(\operatorname{ord}_{t} a_{j}(t)+q(d-j)\right)=\operatorname{ord}_{t} F\left(t, U t^{q}+\xi(t)\right)
$$

for any $\xi \in \mathbb{L}\left(\left(t^{\mathbb{Q}}\right)\right), \operatorname{ord}_{t} \xi(t) \geqslant q$ where $\mathbb{L}$ is an overfield of $\mathbb{K}(U)$. This gives the first part of the lemma.

" $1 \Rightarrow 2 "$ Take any representative $\varphi(t) \in \stackrel{\circ}{\mathscr{J}} q[0]$. Then one can write $\varphi(t)=$ $\sum_{r \geqslant q} \varphi_{r} t^{r}$ with $\varphi_{r} \in \mathbb{K}$, so $v(t):=\left(U-\varphi_{q}\right) t^{q}+\varphi(t)$ is a $(q, U)$-deformation of 0 . By assumption and the first part of the proof, $\operatorname{ord}_{t} F(t, v(t)) \geqslant N$ and substituting $U=\varphi_{q}$ into this relation we obviously get

$$
\operatorname{ord}_{t} F(t, \varphi(t)) \geqslant N
$$

" $2 \Rightarrow 1$ " Let $h(U) t^{\alpha}:=\operatorname{info}_{t} F\left(t, U t^{q}\right)$. Since $h(U) \in \mathbb{K}[U] \backslash\{0\}$ and the field $\mathbb{K}$ is infinite, there exists $x_{0} \in \mathbb{K}$ such that $h\left(x_{0}\right) \neq 0$. This implies that $\operatorname{ord}_{t} F\left(t, x_{0} t^{q}\right)=\alpha$. But $x_{0} t^{q} \in \stackrel{\circ}{J}^{q}[0]$, so by assumption it is $\alpha \geqslant N$.

\subsection{Parametrizations}

In what follows, we will utilize an even broader class of parametrizations than the formal ones (cf. Definition 2). Namely, the following strengthening of Theorem 4 holds true. 
Proposition 2 Let $\mathbb{K}=\overline{\mathbb{K}}$. For any $f \in \mathbb{K}((X))[Y] \operatorname{deg}_{Y} f>0$, there exists a pair $(\varphi(t), \psi(t)) \in \mathbb{K}((t))^{2}$ with $\infty>\operatorname{ord}_{t} \varphi(t)>0$ such that

$$
f(\varphi(t), \psi(t))=0 .
$$

Conversely, for any pair $(\varphi(t), \psi(t))$ as above there exists an irreducible $f \in$ $\mathbb{K}[[X]][Y]$ with $\operatorname{deg}_{Y} f>0$ and such that

$$
f(\varphi(t), \psi(t))=0 .
$$

Proof “ $\Leftarrow$ " Let $(\varphi(t), \psi(t)) \in \mathbb{K}((t))^{2}$ with $\infty>\operatorname{ord}_{t} \varphi(t)>0$. If $\operatorname{ord}_{t} \psi(t)>0$ then the existence of $f$ is a direct consequence of Theorem 4. Similarily, it is easy to treat the case $\operatorname{ord}_{t} \psi(t)=0$. Thus, we may assume that $\operatorname{ord}_{t} \psi(t)<0$ and use Theorem 4 to find a $g \in \mathbb{K}[[X]][Y] \backslash\{0\}$ such that $g(\varphi(t), 1 / \psi(t))=0$. Now it is enough to put $f:=g\left(X, Y^{-1}\right) Y^{a}$, for a big enough $a \in \mathbb{N}$, to assure that $f \in \mathbb{K}[[X]][Y]$ and $f(\varphi(t), \psi(t))=0$. Notice that the latter condition together with $\operatorname{ord}_{t} \varphi(t)<\infty$ imply that necessarily $\operatorname{deg}_{Y} f>0$. Thus, we can factor $f$ in $\mathbb{K}[[X]][Y]$ into irreducible elements and replace $f$ by an irreducible one that also vanishes at $(\varphi(t), \psi(t))$. By the above remark, it has to be $\operatorname{deg}_{Y} f>0$ also for the changed $f$.

“ $\Rightarrow$ " Let $f \in \mathbb{K}((X))[Y] \operatorname{deg}_{Y} f>0$. Write $f=a_{0}(X) Y^{k}+\cdots+a_{k-1}(X) Y+$ $a_{k}(X)$. Note that by considering $f\left(X, X^{\alpha} Y\right)$, where $\alpha \in \mathbb{Q} \leqslant 0$, one can arrange things so that $r:=\min _{0 \leqslant j \leqslant k-1}\left(\operatorname{ord}_{X} a_{j}(X)\right)<\operatorname{ord}_{X} a_{k}(X)$ and then taking $g:=$ $X^{-r} f\left(X, X^{\alpha} Y\right)$ we have $g \in \mathbb{K}[[X]][Y], \operatorname{deg}_{Y} g>0, g(\mathbf{0})=0$ and $X \nmid g$ in $\mathbb{K}[[X, Y]]$. Applying Theorem 4 to $g$ we find a parametrization $\Phi(t)=\left(\Phi_{1}(t), \Phi_{2}(t)\right) \in$ $\mathbb{K}[[t]]^{2} \backslash\{\boldsymbol{0}\}$ of $g$ such that $\operatorname{ord}_{t} \Phi_{1}(t)>0$. Also, $\operatorname{ord}_{t} \Phi_{1}(t)<\infty$, since otherwise $g\left(0, \Phi_{2}(t)\right)=0$, implying $X \mid g$ in $\mathbb{K}[[X, Y]]$. Now it is enough to consider $(\varphi(t), \psi(t)):=\left(\Phi_{1}(t), \Phi_{1}^{\alpha}(t) \cdot \Phi_{2}(t)\right) \in \mathbb{K}((t))^{2}$ to fulfill the needed conditions.

Thus, it is natural to define what follows.

Definition 8 Let $\mathbb{K}$ be a field. Any pair of the form $(\varphi(t), \psi(t)) \in \mathbb{K}((t))^{2}$ with $\infty>\operatorname{ord}_{t} \varphi(t)>0$ will be called a Laurent parametrization. If $f \in \mathbb{K}((X))[Y]$ and $f(\varphi(t), \psi(t))=0$ we will say that such a pair is a Laurent parametrization of $f$. For $f \in \mathbb{K}[X, Y]$ a pair $(\varphi(t), \psi(t)) \in \mathbb{K}((t))^{2}$ with $\operatorname{ord}_{t}(\varphi(t), \psi(t))<0$ and such that $f(\varphi(t), \psi(t))=0$ will be called a parametrization of $f$ at infinity.

The following property is immediate.

Corollary 1 Let $\mathbb{K}=\overline{\mathbb{K}}$. For any $f \in \mathbb{K}[X, Y]$, deg $f>0$, there exists a parametrization of $f$ at infinity.

Proof If $\operatorname{deg}_{Y} f>0$, it is enough to consider $f\left(X^{-1}, Y\right)$ and use Proposition 2 to find $(\varphi(t), \psi(t)) \in \mathbb{K}((t))^{2}$ with $\infty>\operatorname{ord}_{t} \varphi(t)>0$ such that $f\left(\varphi(t)^{-1}, \psi(t)\right)=0$. Similarily for the case $\operatorname{deg}_{X} f>0$.

An important connection between the Laurent and the generalized parametrizations is given in the proposition below. Note that the proof is mainly for the case of a field $\mathbb{K}$ of positive characteristic, since otherwise a standard application of the Implicit Function Theorem suffices. 
Proposition 3 Let $\mathbb{K}=\overline{\mathbb{K}}$. Let $(\varphi(t), \psi(t)) \in \mathbb{K}((t))^{2}$ and $(t, y(t)) \in \mathbb{K}\left(\left(t^{\mathbb{Q}}\right)\right)^{2}$ be a Laurent (resp. a generalized) parametrization of the same irreducible and monic $f \in \mathbb{K}((X))[Y]$. Then for every $g=g(X, Y) \in \mathbb{K}((X))[Y]$ it is

$$
\operatorname{ord}_{t} g(t, y(t))=\frac{\operatorname{ord}_{t} g(\varphi(t), \psi(t))}{\operatorname{ord}_{t} \varphi(t)} .
$$

Proof Consider the field $\mathbb{E}:=\mathbb{K}((t))[Y] /(f(t, Y)) \cong \mathbb{K}((t))(y(t))$. It is a finite extension of the field $\mathbb{F}:=\mathbb{K}((t))$. Define $v_{1}, v_{2}: \mathbb{E} \rightarrow \mathbb{R} \cup\{\infty\}$ by the formulas

$$
v_{1}([g]):=\frac{\operatorname{ord}_{t} g(\varphi(t), \psi(t))}{\operatorname{ord}_{t} \varphi(t)} \quad \text { and } \quad v_{2}([g]):=\operatorname{ord}_{t} g(t, y(t)),
$$

where $g=g(t, Y) \in \mathbb{K}((t))[Y]$. Since both $(\varphi(t), \psi(t))$ and $(t, y(t))$ are parametrizations of $f$, it is easy to see that $v_{1}$ and $v_{2}$ are correctly defined (recall also that by definition $\left.\operatorname{ord}_{t} \varphi(t) \neq 0\right)$.

We claim that $v_{1}, v_{2}$ are valuations on the field $\mathbb{E}$. Indeed, most of the needed conditions follow at once from the corresponding properties of the order function. The only thing worth a closer look is the implication: " $v_{i}([g])=\infty \Rightarrow[g]=0$ ". For $v_{2}$ this is immediate, because $f(t, Y)$ is the minimal polynomial of $y(t)$ over $\mathbb{F}$, so $g(t, y(t))=0$ implies $f(t, Y) \mid g$ in $\mathbb{F}[Y]$. For $v_{1}$, let $g(\varphi(t), \psi(t))=0$ and consider the set $\mathscr{I}:=\{h \in \mathbb{F}[Y]: h(\varphi(t), \psi(t))=0\}$. Obviously, this is an ideal in $\mathbb{F}[Y]$, which itself is a PID, so it is generated by a single element $\tilde{f} \in \mathbb{F}[Y]$. But $f(t, Y)$ is irreducible in $\mathbb{F}[Y]$ and also belongs to $\mathscr{I}$. Hence, $f(t, Y) \sim \tilde{f}$. Since $g \in \mathscr{I}$, we thus conclude that $f(t, Y) \mid g$ and $[g]=0$.

Now, observe that for $h=h(t) \in \mathbb{F}$ it is

$$
v_{1}([h])=\frac{\operatorname{ord}_{t} h(\varphi(t))}{\operatorname{ord}_{t} \varphi(t)}=\operatorname{ord}_{t} h(t)=v_{2}([h]) .
$$

Define $|\cdot|_{i}:=2^{-v_{i}(\cdot)}, i=1,2$. Then $|\cdot|_{1},|\cdot|_{2}$ are two absolute values on the field $\mathbb{E}$, that by (8) agree on the subfield $\mathbb{F}$ with the absolute value $|\cdot|:=2^{-\operatorname{ord}_{t}(\cdot)}$. Since the extension $\mathbb{E} \supset \mathbb{F}$ is finite and $(\mathbb{F},|\cdot|)$ is complete $(|\cdot|$ defines the usual $t$-adic topology on $\mathbb{F}$ ), we can apply [12, Thm. 9.8] to conclude that $|\cdot|_{1}=|\cdot|_{2}$. But this means that also $v_{1}=v_{2}$. Now (6) follows from (7).

Remark 2 Let $\mathbb{K}=\overline{\mathbb{K}}$. It is well-known that if $f, g \in \mathbb{K}[[X, Y]], f(\mathbf{0})=g(\mathbf{0})=0$ and $f$ is irreducible, then the Hilbert-Samuel multiplicity of $(f, g)$ is given by the formula

$$
e(f, g)=\operatorname{ord}_{t} g(\varphi(t), \psi(t))
$$

where $(\varphi(t), \psi(t))$ is a formal parametrization of $f$ (see e.g. [17, Thm. 3.14]). From Proposition 3 it follows that in a generic coordinate system we have 


$$
e(f, g)=\operatorname{ord}_{(X, Y)} f(X, Y) \operatorname{ord}_{t} g(t, y(t))
$$

where $(t, y(t))$ is a generalized parametrization of $f$.

\section{Main results}

We start with a general result concerning the pseudoexponent $\overline{\mathfrak{L}}$. It contains, inter alia, a description of all the jets extracting the pseudoexponent, a result that is inspired by [14], where the classical case of germs of functions (in the real analytic setting) has been considered.

Theorem 5 Let $\mathbb{K}=\overline{\mathbb{K}}$. Then for every tuple $F=\left(f_{1}, \ldots, f_{m}\right) \in \mathbb{K}\left(\left(X^{\mathbb{Q}}\right)\right)[Y]^{m}$ with $\operatorname{deg}_{Y} F>0$ the Eojasiewicz pseudoexponent of $F$ is attained on a generalized parametrization of a component of $F$. Furthermore, if $m \geqslant 2$ and $f_{1}, \ldots, f_{m}$ are coprime (as polynomials), then there exists a set $\stackrel{\mathfrak{J}}{\subset} \bigcup_{q \in \mathbb{Q}} \stackrel{\circ}{\mathscr{J}} q$ of open jets of $\mathbb{K}\left(\left(t^{\mathbb{Q}}\right)\right.$ ) such that:

(a) $\stackrel{\mathfrak{J}}{\mathrm{J}}$ is non-empty and finite,

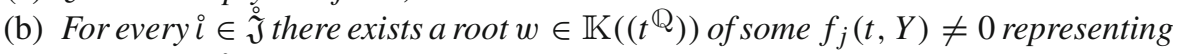
i, i.e. $\stackrel{i}{=} \stackrel{\circ}{\mathscr{J}} q[w]$ for some $q \in \mathbb{Q}$, with

$$
\overline{\mathfrak{L}}(F)=\operatorname{ord}_{t} F(t, w(t))=\operatorname{ord}_{t} F(t, i),
$$

(c) For every $\varphi \in \mathbb{K}\left(\left(t^{\mathbb{Q}}\right)\right)$ it is

$$
\overline{\mathfrak{L}}(F)=\operatorname{ord}_{t} F(t, \varphi(t)) \Leftrightarrow \stackrel{\circ}{\mathscr{J}} q[\varphi] \in \stackrel{\mathfrak{J}}{\text { for some }} q \in \mathbb{Q} .
$$

Proof It is clear that we can assume that $f_{1}(t, Y), \ldots, f_{m}(t, Y)$ have no common root in $\mathbb{K}\left(\left(t^{\mathbb{Q}}\right)\right)$. Also, it is enough to consider the case of all the $f_{j}$ being non-zero. Let $\left\{u_{i j}\right\} \subset \mathbb{K}\left(\left(t^{\mathbb{Q}}\right)\right)$ be the set of all the roots of $f_{1}(t, Y) \times \cdots \times f_{m}(t, Y)$; precisely, let $f_{i}\left(t, u_{i j}(t)\right)=0$ for $1 \leqslant i \leqslant m, 1 \leqslant j \leqslant l_{i}$ (here, possibly, some - but not all $l_{i}=0$ for a constant $f_{i}$ ). Define

$$
\tilde{\mathfrak{L}}:=\sup _{1 \leqslant i \leqslant m}\left(\sup _{1 \leqslant j \leqslant l_{i}} \operatorname{ord}_{t} F\left(t, u_{i j}(t)\right)\right) .
$$

By the assumptions, $-\infty<\tilde{\mathfrak{L}}<\infty$. We claim that $\tilde{\mathfrak{L}}=\overline{\mathfrak{L}}(F)$. Take any $w \in \mathbb{K}\left(\left(t^{\mathbb{Q}}\right)\right)$ different from all the $u_{i j}$ and let $\rho:=\max _{i, j}\left(\operatorname{ord}_{t}\left(w(t)-u_{i j}(t)\right)\right) \in \mathbb{Q}$.

Let $z(t)$ be any $(\rho, U)$-deformation of 0 . It is

$$
\operatorname{info}_{t} f_{i}\left(t, w^{\leqslant \rho}(t)+z(t)\right)=P_{i}(U) t^{\alpha_{i}}
$$

where $P_{i} \in \mathbb{K}[U], \alpha_{i} \in \mathbb{Q}$ and $i=1, \ldots, m$. By Proposition 1 and the definition of $\rho$, the polynomials $P_{1}, \ldots, P_{m}$ do not vanish at $U=0$. Since (9) is valid in particular for $z(t):=U t^{\rho}+\left(w(t)-w^{\leqslant \rho}(t)\right)$, by taking $U=0$ in these equalities we see that

$$
\operatorname{info}_{t} f_{i}(t, w(t))=\ominus t^{\alpha_{i}}, \quad \text { for } i=1, \ldots, m,
$$


- and consequently that -

$$
\operatorname{ord}_{t}(F(t, w(t)))=\min \left(\alpha_{1}, \ldots, \alpha_{m}\right) .
$$

On the other hand, one can take $z(t):=U t^{\rho}+(u(t)-u \leqslant \rho(t))$, where $u(t) \in\left\{u_{i j}(t)\right\}$ is chosen in such a way that $\rho=\operatorname{ord}_{t}(w(t)-u(t))$. Let $u(t)=\sum_{q \in \mathbb{Q}} u_{q} t^{q}$ and $w(t)=\sum_{q \in \mathbb{Q}} w_{q} t^{q}$. Then

$$
w^{\leqslant \rho}(t)+z(t)=w^{\leqslant \rho}(t)-u^{\leqslant \rho}(t)+U t^{\rho}+u(t)=\left(U+w_{\rho}-u_{\rho}\right) t^{\rho}+u(t) .
$$

Thus, (9) takes the form

$$
\operatorname{info}_{t} f_{i}\left(t, u(t)+\left(U+w_{\rho}-u_{\rho}\right) t^{\rho}\right)=P_{i}(U) t^{\alpha_{i}},
$$

so putting $U=u_{\rho}-w_{\rho}$ we conclude that

$$
\operatorname{ord}_{t}\left(f_{i}(t, u(t))\right) \geqslant \alpha_{i}, \text { for } i=1, \ldots, m,
$$

and so

$$
\operatorname{ord}_{t} F(t, u(t)) \geqslant \min \left(\alpha_{1}, \ldots, \alpha_{m}\right) \text {. }
$$

But since $u(t) \in\left\{u_{i j}(t)\right\}$, using $(10,11)$ and the definition of the number $\tilde{\mathfrak{L}}$ we get

$$
\tilde{\mathfrak{L}} \geqslant \operatorname{ord}_{t} F(t, u(t)) \geqslant \operatorname{ord}_{t} F(t, w(t)) .
$$

Now, $w(t)$ was an arbitrary element of $\mathbb{K}\left(\left(t^{\mathbb{Q}}\right)\right) \backslash\left\{u_{i j}(t)\right\}$. Since the resulting inequality clearly holds for $w(t) \in\left\{u_{i j}(t)\right\}$ by the very definition of $\tilde{\mathfrak{L}}$, it holds for any $w(t) \in$ $\mathbb{K}\left(\left(t^{\mathbb{Q}}\right)\right)$. Thus,

$$
\tilde{\mathfrak{L}} \geqslant \overline{\mathfrak{L}}(F) .
$$

Since the other inequality is obvious, the first assertion of the theorem is proved.

Notice also that from the above reasoning one can actually deduce more:

Claim For every $w \in \mathbb{K}\left(\left(t^{\mathbb{Q}}\right)\right) \backslash\left\{u_{i j}\right\}$ such that $\operatorname{ord}_{t} F(t, w(t))=\overline{\mathfrak{L}}(F)$, if $\rho:=$ $\sup \left(\operatorname{ord}_{t}\left(w-u_{i j}\right)\right)$ then $i, j$

$$
\overline{\mathfrak{L}}(F)=\operatorname{ord}_{t} F\left(t, \stackrel{\circ}{J}^{\rho}[w(t)]\right) .
$$

Indeed, by assumption $w \notin\left\{u_{i j}\right\}$. Hence, using the notations of (9), by (12) and (10) we see that in such a case

$$
\begin{aligned}
\overline{\mathfrak{L}}(F) & =\operatorname{ord}_{t} F(t, u(t))=\operatorname{ord}_{t} F(t, w(t))=\min \left(\alpha_{1}, \ldots, \alpha_{m}\right) \\
& =\operatorname{ord}_{t} F\left(t, w^{\leqslant \rho}(t)+z(t)\right),
\end{aligned}
$$


for any $(\rho, U)$-deformation $z(t)$ of 0 . In particular, one can take $z(t):=U t^{\rho}+$ $\left(w(t)-w^{\leqslant \rho}(t)\right)$. By Lemma 2 , for every representative $\varphi$ of $\stackrel{\circ}{\mathscr{J}} \rho[w(t)]$ we have $\operatorname{ord}_{t} F(t, \varphi(t)) \geqslant \overline{\mathfrak{L}}(F)$. Now, the definition of $\overline{\mathfrak{L}}(F)$ implies that (13) holds.

For the rest of the reasoning, let $\mathscr{M}:=\left\{u_{i j}: \operatorname{ord}_{t} F\left(t, u_{i j}(t)\right)=\overline{\mathfrak{L}}(F)\right\}$, and if $w \in \mathscr{M}$ let $q(w):=\min \left\{q \in \mathbb{Q}: \operatorname{ord}_{t} F\left(t, w(t)+U t^{q}\right)=\overline{\mathfrak{L}}(F)\right\}$. Note that by Lemma 1 the number $q(w)$ is properly defined. We define $\stackrel{\mathfrak{J}}{:}=\left\{\stackrel{\circ}{J}^{q(w)}[w]: w \in \mathscr{M}\right\}$.

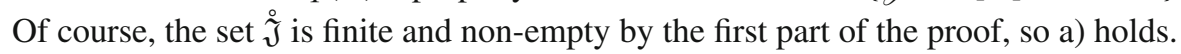

Now, let $i \in \stackrel{\mathfrak{J}}{\text { and let }} w \in \mathscr{M}$ be such that $i=\dot{J}^{(w)}[w]$. Since

$$
\operatorname{ord}_{t} F\left(t, w(t)+U t^{q(w)}\right)=\overline{\mathfrak{L}}(F)
$$

by Lemma 2 and the definition of $\overline{\mathfrak{L}}(F)$ for every representative $\varphi$ of $i$ we have

$$
\operatorname{ord}_{t} F(t, \varphi(t))=\overline{\mathfrak{L}}(F) \text {. }
$$

This proves b).

Considering item c). The implication " $\Leftarrow$ " follows from b). So, assume that $\overline{\mathfrak{L}}(F)=$ $\operatorname{ord}_{t} F(t, \varphi(t))$ for some $\varphi \in \mathbb{K}\left(\left(t^{\mathbb{Q}}\right)\right)$. If $\varphi$ is one of the $u_{i j}$ 's, it belongs to $\mathscr{M}$,

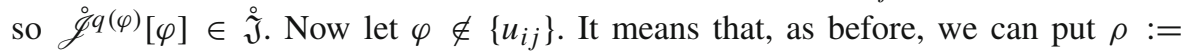
$\sup _{i, j}\left(\operatorname{ord}_{t}\left(\varphi-u_{i j}\right)\right) \in \mathbb{Q}$. Now take any $u \in\left\{u_{i j}\right\}$ such that $\operatorname{ord}_{t}(\varphi-u)=\rho$. Since then $\stackrel{\circ}{J}^{\rho}[\varphi]=\stackrel{\circ}{\mathscr{J}} \rho[u]$, by the Claim we must have

$$
\operatorname{ord}_{t} F(t, \stackrel{\circ}{\rho} \rho[u])=\overline{\mathfrak{L}}(F)
$$

so also $\operatorname{ord}_{t} F(t, u(t))=\overline{\mathfrak{L}}(F)$. In particular, $u \in \mathscr{M}$. Moreover, by Lemma 2 it is also $\operatorname{ord}_{t} F\left(t, u(t)+U t^{\rho}\right)=\overline{\mathfrak{L}}(F)$. Hence, the definition of $q(u)$ implies that $q(u) \leqslant \rho$.

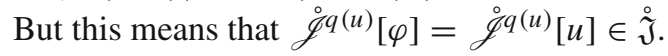

Corollary 2 Let $\mathbb{K}=\overline{\mathbb{K}}$. For every tuple $F=\left(f_{1}, \ldots, f_{m}\right) \in \mathbb{K}\left(\left(X^{\mathbb{Q}}\right)\right)[Y]^{m}$ the pseudoexponent $\overline{\mathfrak{L}}(F)$ is a rational number $($ or $+\infty)$.

Proof If $\operatorname{deg}_{Y} F \leqslant 0$ then $F \in \mathbb{K}\left(\left(X^{\mathbb{Q}}\right)\right)^{m}$ and we have $\overline{\mathfrak{L}}(F)=\sup _{y(t) \in \mathbb{K}\left(\left(t^{\mathbb{Q}}\right)\right)}$ $F(t, y(t))=\operatorname{ord}_{t} F(t) \in \mathbb{Q} \cup\{+\infty\}$. If $\operatorname{deg}_{Y} F>0$ and $f_{1}, \ldots, f_{m}$ are co-prime then by Theorem 5 there exists $w(t) \in \mathbb{K}\left(\left(t^{\mathbb{Q}}\right)\right)$ such that $\overline{\mathfrak{L}}(F)=\operatorname{ord}_{t} F(t, w(t)) \in \mathbb{Q}$. Lastly, if $h \mid f_{j}, j=1, \ldots, m$, where $h \in \mathbb{K}\left(\left(X^{\mathbb{Q}}\right)\right)[Y]$ and $\operatorname{deg}_{Y} h>0$ then by Theorem 3 there exists $y(t) \in \mathbb{K}\left(\left(t^{\mathbb{Q}}\right)\right)$ such that $h(t, y(t))=0$. This gives $\overline{\mathfrak{L}}(F)=$ $+\infty$.

Example 2 Let $\mathbb{K}$ be an algebraically closed field. Consider $F:=\left(Y-\sum_{2 \leqslant q} X^{1-1 / q}-\right.$ $\left.X,\left(Y-\sum_{2 \leqslant q} X^{1-1 / q}\right)^{2}\right) \in \mathbb{K}\left(\left(X^{\mathbb{Q}}\right)\right)[Y]^{2}$. By the theorem, one easily sees that $\overline{\mathfrak{L}}(F)=\max \{1,2\}=2$ and the exponent is realized only by the parametrization of the first component of $F$, that is by $(t, y(t)):=\left(t, \sum_{2 \leqslant q} t^{1-1 / q}+t\right)$. It follows that $q(y)=2$ and $\stackrel{\mathfrak{J}}{=}=\left\{\stackrel{\circ}{\mathscr{J}}^{2}[y]\right\}$ (see the proof of Theorem 5). 
Theorem 6 Let $\mathbb{K}=\overline{\mathbb{K}}$. Then for any $F=\left(f_{1}, \ldots, f_{m}\right) \in \mathbb{K}[[X, Y]]^{m}$, such that $F(\mathbf{0})=\mathbf{0}$, there exists a formal parametrization $\Phi(t)$ of some $f_{j}$ such that

$$
\mathfrak{L}_{0}(F)=\frac{\operatorname{ord}_{t} F(\Phi(t))}{\operatorname{ord}_{t} \Phi(t)} .
$$

Proof We may assume that all $f_{j}$ are non-zero. Moreover, using Weierstrass Preparation Theorem (after possible change of variables) we may assume that for each $f_{j}$ we have $f_{j}(X, Y) \in \mathbb{K}[[X]][Y]$, ord $(X, Y) f_{j}(X, Y)=\operatorname{deg}_{Y} f_{j}(X, Y)>0$. Observe that if $(t, y(t))$ (resp. $(\varphi(t), \psi(t)))$ is a generalized (resp. formal) parametrization of some $f_{j}$ then $\operatorname{ord}_{t} y(t) \geqslant 1\left(\operatorname{resp} . \operatorname{ord}_{t} \psi(t) \geqslant \operatorname{ord}_{t} \varphi(t), \varphi(t) \neq 0\right)$. Thus, by Theorems 4, 5 and Proposition 3 we have

$$
\begin{aligned}
\overline{\mathfrak{L}}(F) & =\sup _{y(t) \in \mathbb{K}\left(\left(t^{\mathbb{Q}}\right)\right)} \operatorname{ord}_{t} F(t, y(t)) \\
& =\sup \left\{\operatorname{ord}_{t} F(t, y(t)):(t, y(t)) \text { is } a \text { generalized parametrization of some } f_{j}\right\} \\
& =\sup \left\{\frac{\operatorname{ord}_{t} F(\varphi(t), \psi(t))}{\operatorname{ord}_{t}(\varphi(t), \psi(t))}:(\varphi(t), \psi(t)) \text { is } a \text { formal parametrization of some } f_{j}\right\} \\
& \leqslant \mathfrak{L}_{0}(F) \leqslant \overline{\mathfrak{L}}(F) .
\end{aligned}
$$

This ends the proof.

Theorem 7 Let $\mathbb{K}=\overline{\mathbb{K}}$. Then for any polynomial map $F=\left(f_{1}, \ldots, f_{m}\right) \in$ $\mathbb{K}[X, Y]^{m}$ there exists a parametrization at infinity $\Phi(t)$ of some $f_{j}$ such that

$$
\mathfrak{L}_{\infty}(F)=\frac{\operatorname{ord}_{t} F(\Phi(t))}{\operatorname{ord}_{t} \Phi(t)} .
$$

Proof We may assume that all $f_{j}$ are non-zero and (after change of variables) that all of them satisfy

$$
\operatorname{deg}_{(X, Y)} f_{j}(X, Y)=\operatorname{deg}_{Y} f_{j}(X, Y)
$$

More specifically,

$$
f_{j}(X, Y)=Y^{d_{j}}+a_{j, 1}(X) Y^{d_{j}-1}+\cdots+a_{j, d_{j}}(X),
$$

where $\operatorname{deg}_{X} a_{j, k}(X) \leqslant k$. Observe that

$$
\begin{aligned}
\mathfrak{L}_{\infty}(F)= & \inf \left\{\frac{\operatorname{ord}_{t} F(\varphi(t), \psi(t))}{\operatorname{ord}_{t} \varphi(t)}: \varphi(t), \psi(t) \in \mathbb{K}((t)),\right. \\
& \left.\operatorname{ord}_{t} \varphi(t) \leqslant \operatorname{ord}_{t} \psi(t), \operatorname{ord}_{t} \varphi(t)<0\right\}
\end{aligned}
$$

and

$$
f_{j}(\varphi(t), \psi(t))=0 \wedge \operatorname{ord}_{t}(\varphi(t), \psi(t))<0 \Rightarrow \operatorname{ord}_{t} \varphi(t) \leqslant \operatorname{ord}_{t} \psi(t)
$$


Let $\tilde{F}=\left(\tilde{f}_{1}, \ldots, \tilde{f}_{m}\right)$, where $\tilde{f}_{j}(X, Y)=f_{j}\left(X^{-1}, Y\right)$. Using Proposition 3 we get $\mathfrak{L}_{\infty}(F) \geqslant-\overline{\mathfrak{L}}(\tilde{F})$. By Theorem 5 there exists $y_{0}(t) \in \mathbb{K}\left(\left(t^{\mathbb{Q}}\right)\right)$ such that $\overline{\mathfrak{L}}(\tilde{F})=$ $\operatorname{ord}_{t} \tilde{F}\left(t, y_{0}(t)\right)$ and $\tilde{f}_{j_{0}}\left(t, y_{0}(t)\right)=0$ for some $j_{0}$. Let (by Propositions 2 and 3 ) $\left(\varphi_{0}(t), \psi_{0}(t)\right) \in \mathbb{K}((t))^{2}$ be a Laurent parametrization of $\tilde{f}_{j_{0}}$ such that for every $g=g(X, Y) \in \mathbb{K}((X))[Y]$ it is

$$
\operatorname{ord}_{t} g\left(t, y_{0}(t)\right)=\frac{\operatorname{ord}_{t} g\left(\varphi_{0}(t), \psi_{0}(t)\right)}{\operatorname{ord}_{t} \varphi_{0}(t)} .
$$

Put $\Phi(t):=\left(1 / \varphi_{0}(t), \psi_{0}(t)\right)$. Since $\operatorname{ord}_{t} \varphi_{0}(t)>0, \Phi(t)$ is a parametrization at infinity of $f_{j_{0}}$. Moreover, by (14) we have $\operatorname{ord}_{t} \Phi=\operatorname{ord}_{t} 1 / \varphi_{0}$. Consequently,

$$
\begin{aligned}
\mathfrak{L}_{\infty}(F) & \geqslant-\overline{\mathfrak{L}}(\tilde{F})=-\operatorname{ord}_{t} \tilde{F}\left(t, y_{0}(t)\right) \\
& =-\frac{\operatorname{ord}_{t} \tilde{F}\left(\varphi_{0}(t), \psi_{0}(t)\right)}{\operatorname{ord}_{t} \varphi_{0}(t)}=\frac{\operatorname{ord}_{t} F(\Phi(t))}{\operatorname{ord}_{t} \Phi(t)} \geqslant \mathfrak{L}_{\infty}(F) .
\end{aligned}
$$

The following two examples demonstrate how to use the above theorems to calculate the Łojasiewicz exponent.

Example 3 A. Płoski in [16] proved that a rational number is equal to the Łojasiewicz exponent of a holomorphic mapping of $\mathbb{C}^{2}$ if and only it appears in the sequence

$$
1,2,3,4,4 \frac{1}{3}, 4 \frac{1}{2}, 4 \frac{2}{3}, 5, \ldots
$$

that is, is a positive integer or of the form $N+\frac{b}{a}$, where $0<b<a<N, a, b, N \in \mathbb{Z}$.

Let $\mathbb{K}$ be an algebraically closed field. We will check that any number from the above sequence is realized as the local Łojasiewicz exponent of some pair $F \in \mathbb{K}[[X, Y]]^{2}$. It is immediate to see from the definition of the local Łojasiewicz exponent that $\mathfrak{L}_{0}\left(X^{N}, Y\right)=N$. Following Płoski, let us consider $F:=\left(Y^{a}-X^{a+1}, X^{N-b} Y^{b}\right)$. Observe that all the formal parametrizations of the second component of $F$ are of the form $(\varphi(t), 0)$ or $(0, \psi(t))$. The first component factors as

$$
Y^{a}-X^{a+1}=\prod_{\substack{\varepsilon^{a}=1 \\ \varepsilon \in \mathbb{K}}}\left(Y-\varepsilon X^{1+\frac{1}{a}}\right)^{\alpha}
$$

for some $\alpha \geqslant 1$, and so by Proposition 3 we may assume that its formal parametrizations are of the form $\left(t^{a}, \varepsilon t^{a+1}\right)$. Since

$$
\frac{\operatorname{ord}_{t} F(\varphi(t), 0)}{\operatorname{ord}_{t}(\varphi(t), 0)}=a+1, \quad \frac{\operatorname{ord}_{t} F(0, \psi(t))}{\operatorname{ord}_{t}(0, \psi(t))}=a, \quad \frac{\operatorname{ord}_{t} F\left(t^{a}, \varepsilon t^{a+1}\right)}{\operatorname{ord}_{t}\left(t^{a}, \varepsilon t^{a+1}\right)}=N+\frac{b}{a}
$$

by Theorem 6 we get that $\mathfrak{L}_{0}(F)=N+\frac{b}{a}$.

Example 4 Let $\mathbb{K}$ be an algebraically closed field. Inspired by [9] let us consider $F:=\left(\left(X+Y^{q}\right)^{p-1}, Y^{p-1}-\left(X+Y^{q}\right)^{p-1} Y^{q-1}\right) \in \mathbb{K}[X, Y]^{2}$, where $p \geqslant 2, q \geqslant 1$, 
$p, q \in \mathbb{Z}$. It is clear that if $\Phi(t):=\left(\varphi_{1}(t), \varphi_{2}(t)\right) \in \mathbb{K}((t))^{2}$ is a parametrization at infinity of the first component of $F$, then $\operatorname{ord}_{t} \varphi_{1}(t), \operatorname{ord}_{t} \varphi_{2}(t)<0$ and hence $\operatorname{ord}_{t} \Phi(t)=\operatorname{ord}_{t} \varphi_{1}(t)$. Consequently,

$$
\frac{\operatorname{ord}_{t} F(\Phi(t))}{\operatorname{ord}_{t} \Phi(t)}=(p-1) \cdot \frac{\operatorname{ord}_{t} \varphi_{2}(t)}{\operatorname{ord}_{t} \varphi_{1}(t)}=\frac{p-1}{q} .
$$

Now, let $\Psi(t):=\left(\psi_{1}(t), \psi_{2}(t)\right) \in \mathbb{K}((t))^{2}$ be a parametrization at infinity of the second component of $F$. If $\Psi(t)=\left(\psi_{1}(t), 0\right)$ then we get $\frac{\operatorname{ord}_{t} F(\Psi(t))}{\operatorname{ord}_{t} \Psi(t)}=p-1$, which is bigger than $\frac{p-1}{q}$ and hence can be discarded for the computation of $\mathfrak{L}_{\infty}(F)$ (cf. Definition 3). It follows that $1-\left(\psi_{1}(t)+\psi_{2}^{q}(t)\right)^{p-1} \psi_{2}^{q-p}(t)=0$ with $\psi_{2} \neq 0$. Using this relation we get $\operatorname{ord}_{t}\left(\psi_{1}(t)+\psi_{2}^{q}(t)\right)=\frac{p-q}{p-1} \cdot \operatorname{ord}_{t} \psi_{2}(t)$, and this for $\operatorname{ord}_{t} \psi_{2}(t)<0$ implies that $\operatorname{ord}_{t}\left(\psi_{1}(t)+\psi_{2}^{q}(t)\right) \geqslant \operatorname{ord}_{t} \psi_{2}(t) \geqslant \operatorname{ord}_{t} \psi_{2}^{q}(t)$, the inequalities being strict if $q>1$. Consequently, we easily see that the only possibilities are

$$
\begin{aligned}
\operatorname{ord}_{t} \Psi(t) & = \begin{cases}\operatorname{ord}_{t} \psi_{1}(t), & \text { if } q>1 \\
\operatorname{ord}_{t} \psi_{2}(t), & \text { if } q=1\end{cases} \\
& =\left\{\begin{array}{ll}
\frac{p-q}{p-1} \cdot \operatorname{ord}_{t} \psi_{2}(t), & \text { if } \operatorname{ord}_{t} \psi_{2}(t) \geqslant 0, q>1 \text { and } p<q \\
q \cdot \operatorname{ord}_{t} \psi_{2}(t), & \text { if } \operatorname{ord}_{t} \psi_{2}(t)<0 \text { and } q>1 \\
\operatorname{ord}_{t} \psi_{2}(t), & \text { if } q=1
\end{array} .\right.
\end{aligned}
$$

Again because of the relation $\Psi$ satisfies, we get

$$
\begin{aligned}
\frac{\operatorname{ord}_{t} F(\Psi(t))}{\operatorname{ord}_{t} \Psi(t)} & =\frac{\operatorname{ord}_{t} \psi_{2}^{p-q}(t)}{\operatorname{ord}_{t} \Psi(t)} \\
& =\left\{\begin{array}{ll}
p-1, & \text { if } \operatorname{ord}_{t} \psi_{2}(t) \geqslant 0, q>1 \text { and } p<q \\
\frac{p-q}{q}, & \text { if } \operatorname{ord}_{t} \psi_{2}(t)<0 \text { and } q>1 \\
p-q, & \text { if } q=1
\end{array} \geqslant \frac{p}{q}-1 .\right.
\end{aligned}
$$

Now, since it is an easy matter to actually find parametrizations $\Psi$ giving equality in the above formula, we conclude by Theorem 7 that

$$
\mathfrak{L}_{\infty}(F)=\min \left\{\frac{p-1}{q}, \frac{p}{q}-1\right\}=\frac{p}{q}-1 .
$$

Similarly, one can check that for $G:=\left(X^{p-q-1} Y^{q+1}, X^{p-q} Y^{q}-1\right) \in \mathbb{K}[X, Y]^{2}$, with $p>q>0, p, q \in \mathbb{Z}$, we have

$$
\mathfrak{L}_{\infty}(G)=-\frac{p}{q} .
$$

Finally, we immediately see that $\mathfrak{L}_{\infty}(X, X Y-1)=-1$. 
Summing up, $\mathfrak{L}_{\infty}$ takes all rational numbers as its values, over any algebraically closed field $\mathbb{K}$.

We end the paper by asking the following:

Question 1 Can Example 3 be strengthened - are the numbers $N+\frac{b}{a}$, where $0<b<$ $a<N, a, b, N \in \mathbb{Z}$, all the possible (finite) Łojasiewicz exponents $\mathfrak{L}_{0}(F)$ that can be realized for $F \in \mathbb{K}[[X, Y]]^{2}$, for every algebraically closed field $\mathbb{K}$ ?

Question 2 Is our definition of the local Łojasiewicz exponent equivalent to Lejeune and Teissier's "integral closure definition" used in [3], or to Płoski's "characteristic polynomial definition" (cf. [16]), for every algebraically closed field $\mathbb{K}$ ?

Open Access This article is distributed under the terms of the Creative Commons Attribution License which permits any use, distribution, and reproduction in any medium, provided the original author(s) and the source are credited.

\section{References}

1. Abhyankar, S.S.: Two notes on formal power series. Proc. Am. Math. Soc. 7, 903-905 (1956)

2. Abhyankar, S.S.: Lectures on expansion techniques in algebraic geometry, volume 57 of Tata Institute of Fundamental Research Lectures on Mathematics and Physics. Tata Institute of Fundamental Research, Bombay, Notes by Balwant Singh (1977)

3. Bivià-Ausina, C., Encinas, S.: Łojasiewicz exponent of families of ideals, Rees mixed multiplicities and Newton filtrations. Rev. Mat. Complut. 26(2), 773-798 (2013)

4. Bochnak, J., Risler, J.-J.: Sur les exposants de Lojasiewicz. Comment. Math. Helv. 50(4), 493-507 (1975)

5. Brzostowski, S.: Non-characteristic approximate roots of polynomials. J. Algebra 343, 143-159 (2011)

6. Antonio, C.: Algebroid curves in positive characteristic. Lecture Notes in Mathematics, vol. 813. Springer, Berlin (1980)

7. Chądzyński, J., Krasiński, T.: Exponent of growth of polynomial mappings of $C^{2}$ into $C^{2}$. In Singularities (Warsaw, 1985), volume 20 of Banach Center Publ., PWN, Warsaw, pp. 147-160 (1988)

8. Chądzyński, J., Krasiński, T.: The Łojasiewicz exponent of an analytic mapping of two complex variables at an isolated zero. In Singularities (Warsaw, 1985), volume 20 of Banach Center Publ., PWN, Warsaw, pp. 139-146 (1988)

9. Chądzyński, J., Krasiński, T.: On the Łojasiewicz exponent at infinity for polynomial mappings of $C^{2}$ into $C^{2}$ and components of polynomial automorphisms of $C^{2}$. Ann. Polon. Math. 57(3), 291-302 (1992)

10. Chądzyński, J., Krasiński, T.: A set on which the local Łojasiewicz exponent is attained. Ann. Polon. Math. 67(3), 297-301 (1997)

11. Chądzyński, J., Krasiński, T.: A set on which the Łojasiewicz exponent at infinity is attained. Ann. Polon. Math. 67(2), 191-197 (1997)

12. Jacobson, N.: Basic algebra. II. W. H. Freeman and Company, New York, second edition (1989)

13. Kedlaya, K.S.: The algebraic closure of the power series field in positive characteristic. Proc. Am. Math. Soc. 129(12), 3461-3470 (2001). (electronic)

14. Kuo, T.C.: Computation of Lojasiewicz exponent of $f(x, y)$. Comment. Math. Helv. 49, 201-213 (1974)

15. Lejeune-Jalabert, M., Teissier, B.: Clôture intégrale des idéaux et équisingularité. Ann. Fac. Sci. Toulouse Math. 17(6), 781-859 (2008)

16. Płoski, A.: Multiplicity and the Łojasiewicz exponent. In Singularities (Warsaw, 1985), volume 20 of Banach Center Publ., PWN, Warsaw, pp. 353-364 (1988)

17. Płoski, A.: Introduction to the local theory of plane algebraic curves. In: Krasiński, T., Spodzieja, S. (eds.) Analytic and Algebraic Geometry, Wydawnictwo Uniwersytetu Łódzkiego, pp. 115-134 (2013)

18. Ribenboim, P.: Fields: algebraically closed and others. Manuscr. Math. 75(2), 115-150 (1992)

19. Spodzieja, S.: The Łojasiewicz exponent of subanalytic sets. Ann. Polon. Math. 87, 247-263 (2005) 